\title{
Application of the Code THEA to the CONDOPT Experiment in SULTAN
}

\author{
L. Bottura, C. Marinucci, and P. Bruzzone
}

\begin{abstract}
The CONDOPT (CONDuctor OPTimization) experiment has been recently completed in SULTAN. The current sharing behavior of $\mathrm{Nb}_{3} \mathrm{Sn}$ samples was assessed as a function of the number of cyclic loads experienced during current sweeps in a $10 \mathrm{~T}$ background field. We present here results of a computer analysis performed with the code THEA ${ }^{\mathrm{TM}}$ (for consistent Thermal, Hydraulic and Electric Analysis) in support of the interpretation of the experimental results. We focus in particular on the critical current and current sharing temperature runs, providing details on the features and effects of current distribution among cable sub-stages.
\end{abstract}

Index Terms-Cable-in-conduit conductors, critical current, current distribution, current sharing.

\section{INTRODUCTION}

C URRENT sharing and distribution in large superconducting cables for high field magnets is a topic of concern that is difficult to address owing to the complex nature of the interaction among the electric and thermal behavior of the cable. Experimental results on large magnets indicated that premature current sharing and resistive voltage development can be a limiting factor for operation [1]. The same result was recently reproduced in the CONDOPT (CONDuctor OPTimization) experiment performed in the SULTAN test facility at CRPP [2]. In the experiment two medium-size $\mathrm{Nb}_{3} \mathrm{Sn}$ cables were subjected to trapezoidal current cycles with maximum current of $15 \mathrm{kA}$ in a transverse background field of $10 \mathrm{~T}$. The voltage-current characteristic of the samples, already significantly degraded with respect to the single strand, worsened due to cycling. In particular, after cycling both samples showed early resistive voltage development and a factor 2 decrease of the exponent $n$, defined from the measured longitudinal electric field $E$ as a function of the cable current $I$ using the power-law:

$$
E=E_{0}\left(\frac{I}{I_{c}}\right)^{n}
$$

where $I_{c}$ is the critical current measured at the electric field $E_{0}$. At the same time the coupling loss drastically decreased, thus indicating a large increase of the interstrand resistance. Details on the experiment and the results are given in [2].

Manuscript received September 24, 2001.

L. Bottura is with CERN, CH-1211 Geneva 23, Switzerland (e-mail: Luca.Bottura@cern.ch).

C. Marinucci and P. Bruzzone are with CRPP, CH-5232 Villigen PSI, Switzerland.

Publisher Item Identifier S 1051-8223(02)04293-8.
In parallel to the increased focus in the experimental acitivity, new computer codes are becoming available for detailed analysis of current distribution and its effects on cable performance. In this paper we report the main outcome of the analysis performed with the code THEA ${ }^{\mathrm{TM}}$ (Thermal, Hydraulic and Electric Analysis) of CryoSoft [3] in support to the interpretation of the experimental results. The main line of investigation pursued here is to examine the conditions under which an increase in interstrand resistance can lead to premature current sharing, resistive voltage development and a decrease of the cable $n$ exponent. A relatively simple model, as described next, has been used to verify behaviors rather than to match experimental results. This choice was made intentionally to avoid that excessive complexity in the simulation conceals the features sought.

\section{SAMPlE CHARACTERISTICS}

We have considered here only one of the two samples used, referred to as SecA in [2]. This cable represents the last-but-one stage of an ITER CS cable [1]. Its geometry and properties are described in [4]. The CONDOPT sample is a hair-pin with helium inlet at the U-bend, at the bottom of the sample, while electric joints and helium outlet are located at the top. The total length is approximately $3 \mathrm{~m}$. The SULTAN split solenoid provides a uniform background field over approximately $50 \mathrm{~cm}$, centered at about $1 \mathrm{~m}$ from the bottom of the sample. Voltage taps (V10V14) measure the longitudinal voltage along the high field region. In addition transverse voltage is measured in the direction of the background field (V77V99, V88V100) and perpendicular to it (V7V9, V8V10) using several voltage taps placed around the jacket downstream from the high field.

\section{A. Cable Geometry}

The cross section of the cable components is taken from [4]. Copper and noncopper cross sections in the strands are corrected for twisting, dividing the un-twisted value by the cosine of the cabling angle $\theta$. The main cable and sample data are reported in Table I for completeness.

\section{B. Strand Data}

The critical currents measured on the strands of the SecA sample [4] can be adequately fitted using the scaling of Summers [5] with the parameters reported in Table I. Strand measurements were performed at an electric field criterion $E_{0}$ of $0.1 \mu \mathrm{V} / \mathrm{cm}$. The exponent $n$ derived using (1) to describe the voltage-current characteristic of the strand is in the range of 30 to 35 . 
TABLE I

CABLE Geometry and Main Properties

\begin{tabular}{|c|c|c|}
\hline length & (m) & 3.05 \\
\hline strand diameter & $(\mathrm{mm})$ & 0.81 \\
\hline cabling pattern & $(-)$ & $3 \times 3 \times 4 \times 4$ \\
\hline cabling pitches & $(\mathrm{mm})$ & $51,76,136,167$ \\
\hline non-copper cross section ${ }^{(1)}$ & $\left(\mathrm{mm}^{2}\right)$ & 29.7 \\
\hline copper cross section ${ }^{(1)}$ & $\left(\mathrm{mm}^{2}\right)$ & 44.5 \\
\hline cosine of cabling angle $\theta$ & $(-)$ & 0.984 \\
\hline jacket cross section & $\left(\mathrm{mm}^{2}\right)$ & 46.4 \\
\hline helium cross section & $\left(\mathrm{mm}^{2}\right)$ & 44.1 \\
\hline void fraction & $(\%)$ & 36.8 \\
\hline cable wetted perimeter & (mm) & 373 \\
\hline jacket wetted perimeter & $(\mathrm{mm})$ & 9.7 \\
\hline hydraulic diameter & $(\mathrm{mm})$ & 0.473 \\
\hline $\mathrm{T}_{\mathrm{c} 0 \mathrm{~m}}^{(2)}$ & $(\mathrm{K})$ & 17.3 \\
\hline $\mathrm{B}_{\mathrm{c} 20 \mathrm{~m}}{ }^{(2)}$ & (T) & 32.7 \\
\hline$C_{0}^{(2)}$ & $\left(\mathrm{AT}^{0.5} / \mathrm{mm}^{2}\right)$ & 6475 \\
\hline copper RRR & $(-)$ & 107 \\
\hline
\end{tabular}

NOTES:

(1) untwisted.

${ }^{(2)}$ fit parameters for $\mathrm{Nb}_{3} \mathrm{Sn}$ critical properties [5].

\section{MODEL}

\section{A. Cable Model}

We have considered in our model only the last-but-one cable stage, modeling the SecA cable as 4 twisted superstrands with uniform properties as derived from Table I. Each superstrand has independent temperature and current. For the calculation of the critical properties we have taken a compressive strain of $-0.68 \%$, consistent with the value expected in a stainless steel jacket. The nonlinear voltage-current characteristic (1) is used in each superstrand. As discussed later, we have taken the exponent $n$ as a matching parameter in the simulations, with an initial value of 15 as measured in the cable in virgin state. In the model the superstrands are thermally coupled through a small thermal resistance. They are also independently cooled by a single helium flow, through heat transfer at the wetted perimeter. Standard correlations (Dittus-Boelter) have been used for the calculation of the heat transfer coefficient. The friction factor of the flow was obtained using the correlation of Katheder [6] adjusted for the void fraction of SecA.

The superstrands are also coupled electrically through mutual inductances and interstrand conductance. We have made the simplifying hypothesis that electric coupling among all superstrands is identical thus neglecting geometric effects on inductance and cable contacts topology. The values used for the reference self and mutual inductances and the interstrand conductance per unit of cable length are given in Table II. The conductance value taken as a reference, 1.2 MS/m, corresponds to the range of interstrand resistance measured among strands in the last-but-one stage of a virgin cable sample [4].

\section{B. Self-Field}

The self-field of the sample $B_{\text {self }}$ can be significant, around $0.4 \mathrm{~T}$ peak in the range of critical currents considered. In the simulations we have approximated the total field $B$ on a super-
TABLE II

COEFFICIENTS OF INDUCTANCE AND CONDUCTANCE AMONG SUPERSTRANDS

\begin{tabular}{lcc}
\hline self inductance & $(\mu \mathrm{H} / \mathrm{m})$ & 1 \\
mutual inductance & $(\mu \mathrm{H} / \mathrm{m})$ & 0.1 \\
conductance & $(\mathrm{MS} / \mathrm{m})$ & 1.2 \\
\hline
\end{tabular}

strand as the sum of the background field, $B_{\mathrm{back}}=10 \mathrm{~T}$, and of a periodically modulated contribution given by

$$
B_{\text {self }}=\Delta B_{\text {self }} \sin \left(\frac{2 \pi x}{L_{p}}+\varphi\right)
$$

where $x$ is the longitudinal coordinate and $L_{p}$ is the twist pitch of the last-but-one cabling stage. The self field amplitude $\Delta B_{\text {self }}$ is taken proportional to the current $I$, with an averaged strength over the last-but-one cabling stage, leading to:

$$
\Delta B_{\text {self }} \approx 0.15 \times 10^{-4} I .
$$

The physical distribution of field in a cable is reproduced shifting the initial phase $\varphi$ by a quarter of a period from superstrand to superstrand.

\section{Joints}

Electrical joints can have a large impact on current sharing results especially in short samples. We have explored the effect of joints parametrically by using simple approximations of ideal conditions. The two conditions considered here are:

- perfect contact among the superstrands, resulting in zero voltage difference among them or also zero joint impedance;

- prescribed current at each superstrand, equivalent to an infinite impedance at the joint.

With this choice it is not necessary to consider the details of a hypothetical joint resistance distribution that in any case cannot be directly measured. Both limits above can be reproduced by the model acting on the electric boundary conditions.

\section{Operating Conditions}

Critical current runs have been simulated reaching an approximate steady state condition of helium massflow, inlet pressure and temperature with no current in the sample and ramping the sample current following the waveform specified for the experiment. Similarly, current sharing temperature runs were simulated reaching an initial steady state at constant current and ramping the inlet temperature thereafter in accordance with the experiment specification.

\section{RESUlts In ViRgin StATE}

\section{A. Simulation of Critical Current Runs}

In a first step in our analysis we have taken care to match the critical current results obtained on the cable in virgin state. For this study we have taken the ideal joint with zero impedance as reference. The effect of the joint model is discussed in SectionV. We have simulated one of the initial critical current runs with steady state massflow of $3 \mathrm{~g} / \mathrm{s}$ and inlet temperature of $4.5 \mathrm{~K}$. With the choice of parameters described above the model reproduces astonishingly well the measured behaviors of the longitu- 
dinal electric field $E_{\text {long }}$ and of the temperature $T$ at the outlet of the high-field zone, shown in Fig. 1. While for the temperature trace the comparison is straightforward, for the longitudinal electric field some additional remarks are necessary. In the simulations, as in the experiment, all superstrands have different potential along the length. We have then taken for the comparison of Fig. 1 the average voltage of all superstrands at the two cross sections where the voltage pick-ups are soldered on the cable jacket.

The voltage difference among superstrands, appearing in the experiment as a transverse electric field $E_{\text {transv }}$, is due to the local build-up of resistive voltage along the superconductor at the locations where the self-field adds to the background field. The order of magnitude of the transverse voltage measured is compared in Fig. 2 to the typical range of simulated voltage differences among superstrands. Both order of magnitude and overall behavior are clearly well represented.

One additional interesting feature that was observed experimentally and is reproduced by the simulation is the fact that the highest transverse electric field is measured in the direction of the background field, i.e., where the field gradient is smallest. In the direction perpendicular to the background field, i.e., where the self-field adds and subtracts to the background producing the strongest field gradient, the measured transverse electric field is negligible. This result is surprising as one usually expects that current transfer, and the associated transverse voltage, takes place in the direction of the largest field gradient.

A snapshot of the voltage difference $\Delta V$ between two facing superstrands is shown in Fig. 3, together with the total field pattern experienced by one of them (the field seen by the other is the mirror image of the one plotted). We see that, as in the experiment, there is a phase lag between the peak in $B$ and the $\Delta V$ between facing superstrands. The transverse voltage peaks at the locations where $B$ is equal to the background value $B_{\text {back, }}$, i.e., in the direction of $B_{\mathrm{back}}$, while it is zero at the peaks (and wells) of the magnetic field $B$, i.e., in the direction perpendicular to $B_{\text {back. }}$. To explain this effect we remark that the characteristic length necessary for current distribution (of the order of $1 \mathrm{~m}$ for the conditions simulated) is much larger than the cable twist pitch. As a result the current in a cable substage is in practice constant along the high field region and any voltage difference among substages can be sustained over a considerable length without significant reduction. The longitudinal voltage can then build-up along the length as the cable substage experiences the region of high field, leading to an analogous increase of the voltage difference with respect to the facing cable substage and thus explaining the peak in $\Delta V$ observed at the end of the high field part.

\section{B. Simulation of Current Sharing Temperature Runs}

The results of simulations performed on current sharing runs at $14.8 \mathrm{kA}$ and $12.7 \mathrm{kA}$ are shown in Fig. 4 . We plot there the longitudinal electric field as a function of the temperature at the outlet of the high field region. For the run at $14.8 \mathrm{kA}$ the simulation still agrees reasonably well with the measurement. For the $12.7 \mathrm{kA}$ run there is an evident discrepancy between measurements and simulation. In general we have found that the disagreement between simulation and measurement increases as a

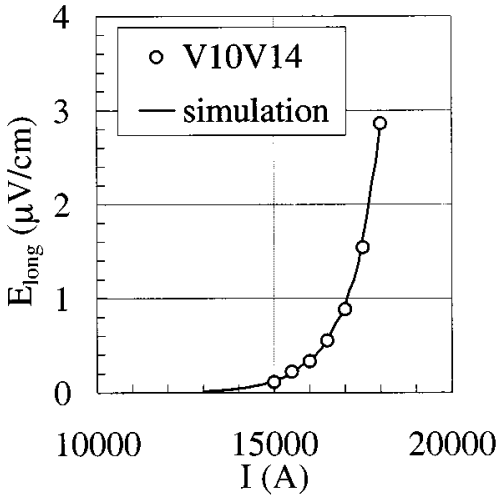

(a)

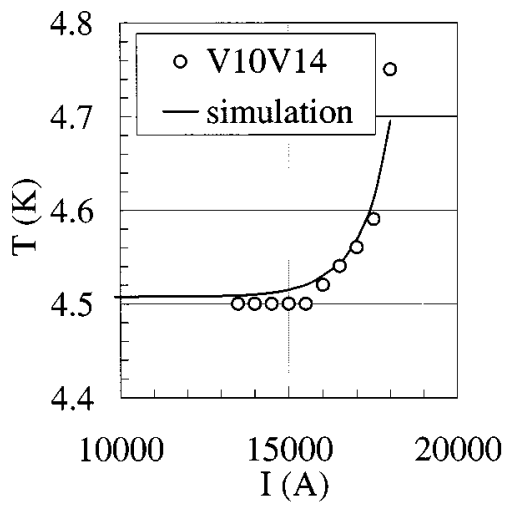

(b)

Fig. 1. Measured and simulated longitudinal electric field (a) and temperature at the outlet of the high field region (b) for the sample SecA in virgin state during a critical current run.

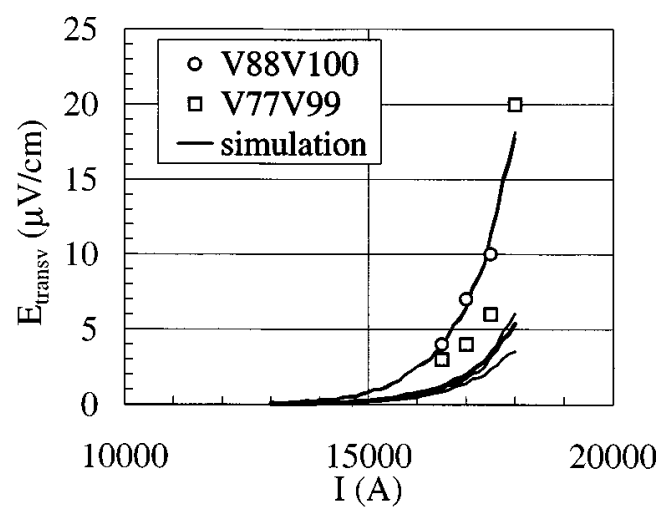

Fig. 2. Comparison of measured and simulated transverse electric field for the conditions of Fig. 1. All simulated voltage differences among the four superstrands at the two locations where the voltage taps are placed on the sample are shown to demonstrate the typical range of transverse voltage.

function of the distance from the well matched reference operating conditions described above. Good agreement can be recovered also in the case of the run at $12.7 \mathrm{kA}$ by artificially choosing a different exponent $n$ in the power law (1). This choice cannot be justified based on physical arguments. We will come back to this point in the discussion.

\section{RESUlTS AFTER CYCLING}

As we anticipated, one of the main results of the experiment was the observation of change in the voltage-current character- 


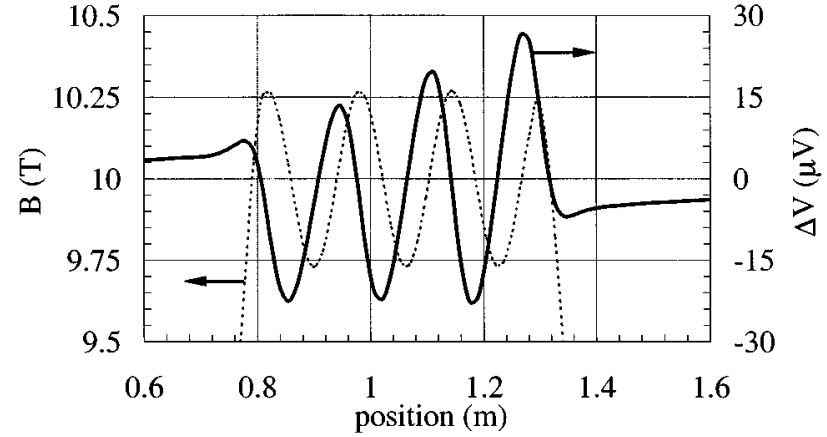

Fig. 3. Simulated voltage difference (transverse voltage $\Delta V$ ) among superstrands 2 and 4, in topologically opposite positions in the model of the cable. The self-field on superstrand 2 is shown to demonstrate the phase relation with the peaks in transverse voltage.

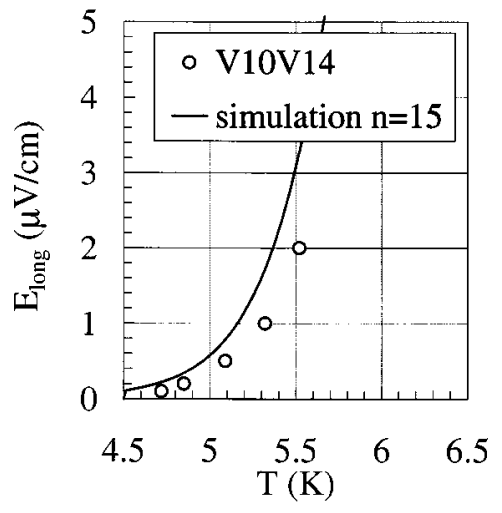

(a)

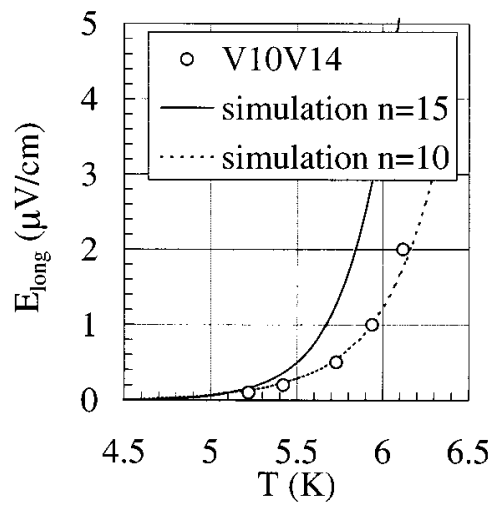

(b)

Fig. 4. Measured and simulated longitudinal electric field as a function of temperature at the end of the high field region during a current sharing temperature run at $14.8 \mathrm{kA}$ (a) and $12.7 \mathrm{kA}$ (b) before cycling.

istics of the sample in concurrence with the increase of the interstrand resistance. We have tested this effect in the model, by decreasing the interstrand conductance by a factor 10 (i.e., down to $0.12 \mathrm{MS} / \mathrm{m}$ ). Simulating the sample with the zero-impedance joint this variation has in practice no effect on the results presented so far. In particular the longitudinal and transverse electric fields are unaffected as can be seen in Fig. 5. The reason is that already with the initial value of interstrand conductance the current transfer takes place over the whole sample length, and at the simulated joint. Decreasing the interstrand conductance only results in a further increase of the current transfer length.

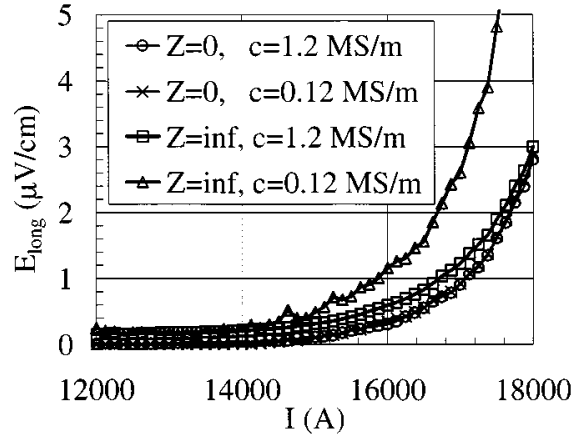

Fig. 5. Simulated longitudinal electric field as a function of current for a critical current run at $3 \mathrm{~g} / \mathrm{s}$ massflow and $4.5 \mathrm{~K}$ inlet temperature. The plot compares results obtained for a joint with either zero or infinite impedance $Z$, and for the nominal and reduced interstrand conductance $c$.

We have then modeled one of the two ends of the sample as a joint with infinite impedance, and a current imbalance of the order of $\pm 8 \%$ which is within the estimated spread of joint resistance. The results of the simulation of a critical current run with $3 \mathrm{~g} / \mathrm{s}$ massflow and $4.5 \mathrm{~K}$ inlet temperature with nominal $(1.2 \mathrm{MS} / \mathrm{m})$ and reduced $(0.12 \mathrm{MS} / \mathrm{m})$ interstrand resistance are reported in Fig. 5. Compared to the results obtained with the zero impedance joint a first effect to be noticed is that already with the nominal interstrand conductance a high joint impedance causes earlier longitudinal voltage development. This effect is due to the transverse voltage appearing along the cable once the current distribution imposed by the joint is forced to change under the longitudinal voltage generated in the high field region. Furthermore, as shown in Fig. 5, the additional voltage associated with this current redistribution grows significantly if the interstrand conductance is decreased. At the same time the temperature at the outlet of the high field region (not shown) increases. The thermal runaway, however, is not significantly affected, and the cable seems to be able to sustain a higher resistive voltage.

\section{DISCUSSION AND CONCLUSION}

The exponent $n$ conveniently used to characterize the voltage-current characteristics of a cable is a good measurement of the collective behavior of the cabled strands. This statement is supported by the fact that once $n$ is known a relatively simple model such as the one used here gives a good interpretation of the behavior of local quantities, such as voltage differences, as well as global quantities, such as cable temperature. On the other hand the value of $n$ is dependent on the operating conditions. We interpret this fact as the indication of an underlying mechanism affecting the voltage-current characteristic of a bundle of cabled strands. We have shown by analysis that this mechanism cannot be the magnetic field gradient or transverse voltage alone. Similarly the variations of interstrand conductance does not explain by itself the changes observed on the $n$ exponent. A possible explanation can be however found postulating that one or more locations in the cable have a longitudinal impedance comparable or larger than the equivalent resistance appearing in the high field region. Candidates for these impedance sources could be the resistance scatter at the joints, with large values on few strands, or local 
current sharing along the cable, such as in the return leg of the sample itself or damaged strands.

\section{REFERENCES}

[1] T. Kato et al., "First test results of the ITER central solenoid model coil," Fusion Engineering and Devices, 2001, to be published.

[2] P. Bruzzone, A. M. Fuchs, B. Stepanov, and G. Vecsey, "Performance evolution of $\mathrm{Nb}_{3} \mathrm{Sn}$ cable-in-conduit conductors under cyclic load," in 17th Int. Magnet Tech. Conf., Geneva, 2001, to be published.
[3] L. Bottura, C. Rosso, and M. Breschi, "A general model for thermal, hydraulic and electric analysis of superconducting cables," Cryogenics, vol. 40, pp. 617-626, 2000.

[4] P. Bruzzone, Ed., "Segregated copper ratio experiment on transient stability (SeCRETS) final report,", CRPP Internal Report LRP 689/01.

[5] L. T. Summers, M. W. Guinan, J. R. Miller, and P. A. Hahn, "A model for the prediction of $\mathrm{Nb}_{3} \mathrm{Sn}$ critical current as a function of field, temperature, strain and radiation damage," IEEE Trans. Magnetics, vol. 27, no. 2, pp. 2041-2044, 1991.

[6] H. Katheder, "Optimum thermohydraulic operation regime for cable in conduit superconductors (CICS)," Cryogenics, vol. 34, pp. 595-598, 1994. 